\title{
Postmortem pathological changes in extrapulmonary organs in SARS-CoV-2 rt-PCR-positive cases: a single-center experience
}

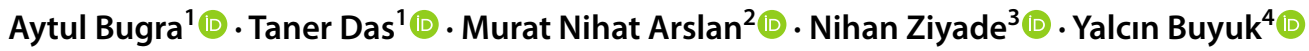

Received: 29 March 2021 / Accepted: 1 May 2021 / Published online: 7 May 2021

(c) Royal Academy of Medicine in Ireland 2021

\begin{abstract}
Background Although the lung is seen as the main target organ affected by SARS-CoV-2, other organs are also damaged. Aim We aimed to determine the extrapulmonary findings of autopsies performed on cases with positive results with postmortem polymerase chain reaction test.

Methods Pathological changes in extrapulmonary organs were examined with light microscopy.

Results Heart, liver, spleen, kidney, pancreas, and central nervous system samples of these cases were evaluated. About $80 \%$ of the cases were men, and $20 \%$ were women. In the examination of heart, 28 of the cases had scar, 14 had acute myocardial infarction, 6 had acute and previous myocardial infarction findings, 2 had myocarditis, and 4 had interstitial mononuclear inflammatory cell infiltration. In the examination of the liver, portal inflammation was observed in 84 of the cases, steatosis in 54, centrilobular necrosis in 9, and capillary endotheliitis in the portal area in 7 of them. In the evaluation of the kidney, 37 cases had chronic pyelonephritis, 36 had tubular damage, 15 had tubulointerstitial necrosis, 16 had subcapsular microhemorrhage, 10 had capillary endothelitis, and 9 had a microvascular fibrin trombosis in their glomerular capillaries. In the central nervous system, 8 cases had infarction and liquefaction, 56 had perivascular petechial hemorrhage, 54 had acute hypoxic ischemic change, 3 had parenchymal microhemorrhage, and 52 had capillary endotheliitis.

Conclusion Autopsies play an important role in systematically examining the damage caused by the virus in all organs in order to elucidate the pathogenesis of SARS-CoV-2 infection and contribute to the clinical management of infected patients.
\end{abstract}

Keywords Autopsy $\cdot$ COVID-19 $\cdot$ Endotheliitis $\cdot$ Pathology $\cdot$ Postmortem $\cdot$ SARS-CoV-2 $\cdot$ Thrombosis

\section{Introduction}

Aytul Bugra

aytulsargan@gmail.com

Taner Das

tanerdas@hotmail.com

Murat Nihat Arslan

mnarslan@yahoo.com

Nihan Ziyade

nihanziyade@gmail.com

Yalcın Buyuk

doctorbuyuk@gmail.com

1 Morgue Department, Histopathology Unit, Council of Forensic Medicine, Istanbul, Turkey

2 Morgue Department, Autopsy Unit, Council of Forensic Medicine, Istanbul, Turkey

3 Morgue Department, Postmortem Microbiology Laboratory, Council of Forensic Medicine, Istanbul, Turkey

4 Council of Forensic Medicine, Istanbul, Turkey
Coronavirus disease-19 (COVID-19), first appeared in the Wuhan region of China, was resulted in a life-threatening pandemic all over the World [1]. As of February 1, 2021, more than 100 million people have been infected and more than 2 million deaths have occurred worldwide [2]. A pandemic was declared by the World Health Organization on March 11, 2020, and the first case was seen in our country on the same date [3].

The Coronavirus family is an enveloped, helical symmetry, positive-polar RNA virus family that causes disease in mammals and birds. It causes respiratory tract infections ranging from a mild cold to pneumonia and rarely gastrointestinal complaints in humans [4]. Although the history of coronaviruses began in the 1940s, the first human coronaviruses, $\mathrm{HCoV} 229 \mathrm{E}$, and $\mathrm{HCoV}-\mathrm{OC} 43$, were reported in the 1960s. After the 2000s, HKU1, HCoV-NL63, SARS$\mathrm{CoV}$, and MERS-CoV have been shown as other members 
of coronaviruses that infect humans [5]. The first four CoVs mentioned are universally constantly in circulation and are causative agents of about one-third of common colds in humans. SARS-CoV and MERS-CoV differ from other coronaviruses in that they cause severe lung infection and the fatality rates are $10 \%$ and $34 \%$, respectively [6].

The causative agent of COVID-19 is a new enveloped RNA virus named "Severe Acute Respiratory Syndrome Coronavirus 2" (SARS-CoV-2) [7]. The source of this new virus has been recognized as bats. First, it gained the property of being transmitted to humans by the zoonotic transition, and then from person to person [8].

SARS-CoV-2 has four main structural proteins: surface glycoprotein (S protein) with rod-like protrusions (spike), small envelope protein, matrix protein, and nucleocapsid protein [9]. The $\mathrm{S}$ protein enables the virus to bind to the cell membrane by interacting with the Angiotensin Converting Enzyme 2 (ACE 2) receptor. ACE 2 receptor has been identified in many organs such as the respiratory system, gastrointestinal system, lymph nodes, thymus, spleen, bone marrow, liver, kidney, and brain [10].

The SARS-CoV-2 infection causes significant proinflammatory cytokine release, exacerbating interstitial pneumonia and acute respiratory distress syndrome. It turns into viral sepsis with hypercoagulation and multiorgan dysfunction [11].

The mortality rate of COVID-19 worldwide is $2 \%$, and $0.06 \%$ of cases are severe. In our country, the mortality rate is $0.9 \%$ and the rate of severe course is $0.7 \%$.

Autopsies of people who died from the SARS-CoV-2 infection can provide important information about the new disease and its course. Autopsies are also important for the statistical recording of deaths of cases detected by postmortem sampling, such as bodies being found dead at home, sudden unexpected deaths, or traumatic deaths, for which SARS-CoV-2 positivity was not known before.

Our study provides a systematic overview of autopsies performed on 100 cases with SARS-CoV-2 positivity at Council of Forensic Medicine, Istanbul Morgue Department. Many studies on SARS-CoV-2 and COVID-19 infection causes have generally focused on lung findings, while studies on extrapulmonary organ findings are limited. We believe that our study provides critical details on pathological changes in extrapulmonary organs since all autopsies were performed with a systematic approach in which all three body cavities were opened.

\section{Materials and methods}

This study was approved by the TR Ministry of Health General Directorate of Health Services Scientific Research Platform (2020-11-22T22_28_27) and the Council of
Forensic Medicine, Education and Scientific Research Commission (21589509/2020/1112-01.12.2020).

The study was perfomed at the Council of Forensic Medicine between April 2020 and February 2021. The study consisted of 100 autopsy cases which were positive for the postmortem SARS-CoV-2 test. A complete autopsy protocol was applied in all cases. All body cavities were opened in a systematic approach with an appropriate autopsy facility for high-risk cases performed under negative pressure environment were by organs and tissues were examined in detail.

Cases with positive postmortem SARS-CoV-2 PCR test applied to swab samples and tissue blocks were included in the study. Cases with negative results in postmortem swab samples were not included in the study.

\section{Autopsy technique}

During the COVID-19 pandemic, in situ autopsy technique was modified and used in Morgue Department Council of Forensic Medicine. All autopsies were carried out by opening three body cavities. Unlike the standard autopsy technique, in minimally invasive autopsy technique, no organ or system was eviscerated except for central nervous system and heart that varies according to the case. Non-eviscerated organs were evaluated by an in situ technique. Samples were taken from all cases for toxicological (blood, intraocular fluid, bile, urine, liver, kidney, stomach contents) and histopathological (brain, cerebellum, brainstem, heart, lung, liver, kidney) examinations in line with standard autopsy procedures. Autopsies were evaluated at the initial examination and triage stage in line with the guidelines published by the Ministry of Health Scientific Board. Nasopharyngeal swab samples were taken before the autopsy from all cases with possible case criteria in their medical history. Tracheal swab and lung swab samples were taken during autopsy from the cases that were not considered to be in the risky group in the pre-autopsy evaluation but raised suspicion in the macroscopic examination of the lungs during the autopsy.

\section{SARS-CoV2 RNA Real Time PCR}

In order to determine SARS-Cov-2 in nasopharyngeal/ tracheal swab samples and paraffin embedded lung tissue samples, real-time RT-PCR method, which is a nucleic acid amplification method that detects viral RNA, was applied. Nucleic acids were extracted on the QIASymphony (Qiagen / Germany) device using the "QIAsymphony DSP Virus/ Pathogen midi" kit. "RealStar® SARS-CoV-2 RT-PCR Kit RUO (Altona Diagnostics, Hamburg, Germany)" was used by RT-PCR method and amplified in Rotor Gene (Qiagen/ Germany) device in accordance with the manufacturer's recommendations. 
In addition, nucleic acids were extracted from all paraffin-embedded lung tissues fixed with formalin. Three or four 10-mm-thick sections of each block were cut by a microtome. The xylene and ethyl alcohol deparaffinization procedure was applied to paraffin-embedded lung tissues. It was then incubated for $24 \mathrm{~h}$ by adding $20 \mathrm{ml}$ of proteinase $\mathrm{K}$ and $600 \mathrm{ml}$ of ATL buffer solution.

\section{Histopathological examination}

Pathological changes in extrapulmonary organs were examined with light microscopy in hematoxylin and eosin stained preparations. Heart, liver, kidney, spleen, pancreas, brain, cerebellum, and brainstem and vascular changes were examined. Lymphocytes were evaluated by CD3 and CD20 immunohistochemical staining methods.

\section{Statistical analysis}

SPSS (Statistical Package for the Social Sciences) V21 2012 program was used for statistical analysis. While evaluating the study data, descriptive statistical methods (average, standard deviation, median, frequency, ratio, minimum, maximum), as well as the chi-square test, were used to compare qualitative data. Significance was evaluated at the $p<0.05$ level.

\section{Results}

About $80 \%$ of the cases were male $(n=80) ; 20 \%$ were female $(n=20)$. The mean age was $54.8 \pm 18.9$ (7-98), and the mean body mass index (BMI) was $28.9 \pm 7.4$ (Table 1). When we look at the distribution of cases according to decades, it was striking that the number of cases were highest in the seventh decade. When evaluated according to gender, the mean age in women was $55.05 \pm 20.8$, and the average age in men was $54 \pm 18.5$. Mean BMI was $32.5 \pm 9.7$ in women and $28 \pm 6.5$ in men. BMI was significantly higher in females than males $(p<0.05)$. Postmortem SARS-CoV-2 PCR test was performed in all cases. Samples that were studied with a nasopharyngeal swab, deep tracheal swab, lung swabs, and sections were taken from paraffin blocks

Table 1 Distribution of age, sex, height, weight and BMI of the cases

\begin{tabular}{lll}
\hline Sex & & \\
Male & $\mathrm{n} / \mathrm{N}$ & $80 / 100$ \\
Female & & $20 / 100$ \\
Age, year & Mean \pm SD (min-max) & $54.8 \pm 18.9(7-98)$ \\
Height, cm & Mean \pm SD (min-max) & $169.9 \pm 10.3(125-189)$ \\
Weight, kg & Mean \pm SD (min-max) & $83.4 \pm 20.8(34-150)$ \\
BMI, $\mathrm{kg} / \mathrm{m}^{2}$ & Mean \pm SD (min-max) & $28,9 \pm 7,4(14.0-55.1)$ \\
\hline
\end{tabular}

from cases suspected histopathologically for COVID-19 (Table 2). A total of 35 cases were found dead, 25 cases died in hospital during the treatment process for illness, 20 cases died suddenly, six cases died after falling from height, five cases died after traffic accident, and eight cases were grouped as other death causes. In one case, no information was available. Considering the causes of death after the autopsy, 74 cases were due to COVID-19 and 26 cases were death accompanied by SARS-CoV-2 positivity. When pathological changes in organs were compared in these cases, no significant difference was found between the two groups $(p>0.05)$. There was hospitalization in 42 cases.

Heart, lung, liver, kidney, brain, cerebellum, and brainstem were sampled for routine histopathological examination in our department. In some cases, the spleen, pancreas, prostate, and small intestine were sampled.

\section{Pathological changes in the heart and coronary arteries}

In all cases, samples were taken from the left ventricular wall, interventricular septum, and right ventricular wall of the heart. The left main coronary artery, the anterior descending branch of the left coronary artery, the circumflex branch of the left coronary artery, and the right coronary artery were also sampled and examined.

Scar, perivascular and interstitial fibrosis, myocardial infarction were the most common lesions in the microscopic examination of the heart. Myocarditis with lymphohistiocytic inflammatory cell infiltration and individual myocyte necrosis was detected in $2 \%(n=2)$ of the cases. In $4 \%$ $(n=4)$ of the cases, mononuclear inflammatory cell infiltration rich in $\mathrm{CD} 3(+)$ lymphocytes was observed in the interstitium. Myocyte injury was not observed in these cases. Acute myocardial infarction was detected in all cases with fibrin thrombosis in their coronary artery. In 5\% $(n=5)$ of these cases, coronary atheroma plaque and fibrin thrombosis were present in the coronary artery. In $1 \%(n=1)$ of the

Table 2 Sampling methods for postmortem Sars-CoV-2 PCR test

\begin{tabular}{ll}
\hline Sampling method & $n / N$ \\
\hline Nasopharyngeal swab & $25 / 100$ \\
Tracheal swab & $29 / 100$ \\
Lung swab & $2 / 100$ \\
Tissue blocks & $6 / 100$ \\
Nasopharyngeal + tracheal & $27 / 100$ \\
Tracheal + lung swab & $3 / 100$ \\
Nasopharyngeal + tracheal + lung Swab & $3 / 100$ \\
Tracheal + lung swab + tissue block & $1 / 100$ \\
Nasopharyngeal swab + tissue block & $3 / 100$ \\
Nasopharyngeal + tracheal + tissue block & $1 / 100$ \\
\hline
\end{tabular}




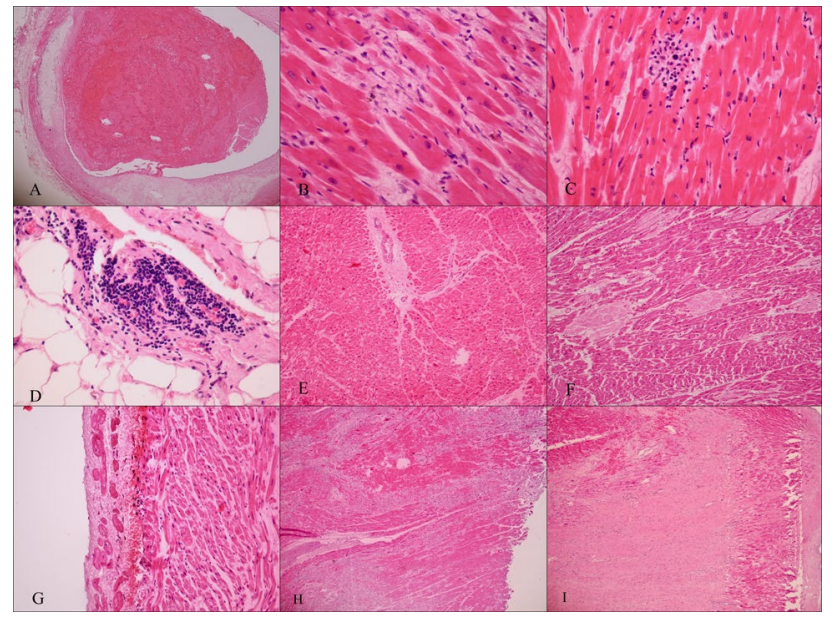

Fig. 1 A Coronary artery atheroma plaque and fibrin thrombosis, $\times$ 40; B Lymphohistiocytic myocarditis, $\times$ 400; C Interstitial mononuclear cells, $\times$ 400; D Lymphocytic pericarditis, $\times$ 400; $\mathbf{E}$ Perivascular and interstitial fibrosis, $\times 100 ;$ F Amyloidosis $\times$ 200; G Subendocardial hemorrhage, $\times 200$; H Myocardial infarction with granulation tissue, $\times 40$; I Scarr tissue in the papillary muscle, $\times 40$, $\mathrm{H} \& \mathrm{E}$

cases, isolated fibrin thrombosis was observed without atheroma plaque. Acute myocardial infarction without thrombosis in the coronary arteries was detected in $4 \%(n=4)$ of the cases. About $9 \%(n=9)$ of the cases could not be optimally evaluated due to autolysis (Fig. 1). Changes related to cardiac pathological findings are given in detail in Table 3.

\section{Pathological Changes in the Liver}

Ninety eight of the 100 cases autopsied had liver samples. Portal and lobular inflammation, macrovesicular steatosis, and centrilobular congestion were among the most common changes observed in the liver. Portal and lobular inflammation were divided into three groups as mild, moderate, and severe. Inflammation rich in CD3 (+) lymphocytes was also observed in the liver. Macrovesicular steatosis was divided into three groups as mild, moderate, and severe, in addition, according to the distribution pattern of steatosis, centrilobular and panacinar subgroups. Periportal steatosis was not observed. Centrilobular mild macrovesicular steatosis was the most common finding. Microvesicular steatosis was seen in 3\% $(n=3)$ of the cases. Centrilobular necrosis was detected in $9 \%(n=9)$ of the cases, capillary endotheliitis in 7\% $(n=7)$, and fibrin thrombus in the portal vein lumen in the portal area in $2 \%(n=2)$ of the cases. Periportal granuloma was seen in $1 \%(n=1)$ of the cases. About $18 \%$ $(n=18)$ of the cases could not be evaluated due to autolysis (Fig. 2). Changes related to pathological findings in the liver are given in detail in Tables 4 and 5 .

\section{Pathological changes in the kidney}

The most common findings in the evaluation of 100 kidney specimens were peritubular and glomerular capillary congestion, chronic pyelonephritis, and tubular damage. Acute tubular necrosis were present in 3\% $(n=3)$ of the cases, interstitial lymphocytic inflammation in $15 \%(n=15)$, subcapsular microhemorrhage in $16 \%(n=16)$, capillary endotheliitis in $11 \%(n=11)$, fibrin thrombosis in glomerular capillaries in 9\% $(n=9)$, fibrin thrombosis in the arcuate branch of the renal artery in $1 \%(n=1)$ of the cases. Autolytic changes were present in $61 \%(n=61)$ of the cases (Fig. 3). Changes related to pathological findings in the kidney are given in detail in Table 6.

\section{Pathological changes in the central nervous system}

Perivascular petechial hemorrhage, acute hypoxicischemic changes, and capillary endothelitiis were the most common findings in the evaluation of 100 brains, cerebellum, and brainstem samples. Most of the cases with subarachnoid and intracranial hemorrhage were due to trauma. Subarachnoid hemorrhage and intracranial hemorrhage due to pathological brain hemorrhage was observed in $1 \%(n=1)$ of the cases. Fibrin thrombosis was also observed in the anterior communicate artery of this case. Pituitary necrosis was seen after cesarean
Table 3 Cardiac pathological findings

\begin{tabular}{llll}
\hline Histopathology & $n / \mathrm{N}$ & Histopathology & $n / N$ \\
\hline Ischemic findings & $47 / 100$ & Lymphohistiocytic Myocarditis & $2 / 100$ \\
Scar & $28 / 100$ & Lymphocytic pericarditis & $8 / 100$ \\
Acute myocardial infarction & $14 / 100$ & Interstitial mononuclear cells & $4 / 100$ \\
Scar+ Acute myocardial infarction & $6 / 100$ & Amyloidosis & $1 / 100$ \\
Perivascular and interstitial fibrosis & $62 / 100$ & Subendocardial hemorrhage & $2 / 100$ \\
Mural thrombosis & $1 / 100$ & Autolysis & $9 / 100$ \\
Coronary artery findings & & & \\
$\quad$ Atheroma plaque & $65 / 100$ & Atheroma plaque + Fibrin thrombosis & $5 / 100$ \\
$\quad$ Normal & $29 / 100$ & Isolated fibrin thombosis & $1 / 100$ \\
\hline
\end{tabular}




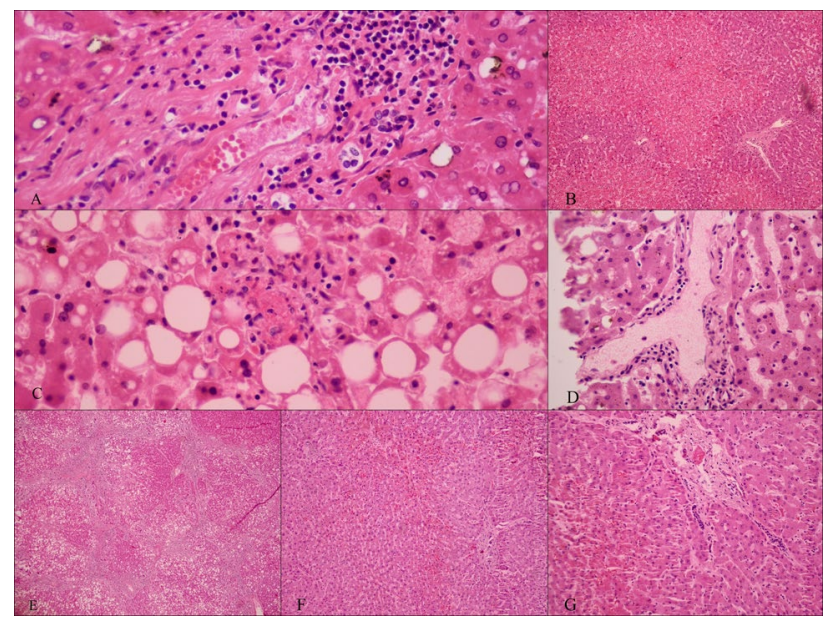

Fig. 2 A Portal inflammation, macrovesicular ve microvesicular steatosis, $\times$ 400; B Centrilobular necrosis, $\times 100$; C Lobular inflammation, $\times$ 400; D Capillary endoteliitis, $\times$ 400; E Cirrhosis, $\times 40 ; \mathbf{F}$ Centrilobular congestion, cholestasis; $\times 100$; G Microvascular thrombosis, $\times 200, \mathrm{H} \& \mathrm{E}$

in a pregnant woman who was positive for SARS-CoV-2 (Fig. 4). Changes related to pathological findings in the central nervous system are given in detail in Table 7.

\section{Pathological changes in the other organs}

Spleen was sampled in 17 cases in 100 autopsies. In 16 of these cases, atrophic changes and PMN leukocyte discharge in the red pulp were observed (Fig. 5).

The pancreas was sampled in 21 cases. About $14 \%(n=3)$ of them had chronic pancreatitis, and 5\% $(n=1)$ had focal acute pancreatitis. About $33 \%(n=7)$ of the cases could not be evaluated due to autolysis.

In one case, the stomach, small intestine, and large intestine were sampled because of the hemorrhagic appearance. Necrotic changes in the epithelium of the small intestine and stomach, active chronic inflammation with membrane-like fibrinous exudate in the mucosa were observed. Intense lymphocytic infiltration was noted around the capillary vessels

Table 4 Hepatic pathological findings

\begin{tabular}{llll}
\hline Histopathology & $n / N$ & Histopathology & $n / N$ \\
\hline Inflammation & $84 / 98$ & Binucleation & $8 / 98$ \\
Steatosis & $54 / 98$ & Glycogenisation & $19 / 98$ \\
Centilobulary necrosis & $9 / 98$ & Autolysis & $18 / 98$ \\
Centrilobulary congestion & $45 / 98$ & Entotheliitis & $7 / 98$ \\
Chirosis & $1 / 98$ & Fibrin thrombosis & $2 / 98$ \\
Bridging necrosis & $2 / 98$ & Granulomatous inflam- & $1 / 98$ \\
Cholestasis & \multicolumn{2}{c}{ mation } \\
\hline
\end{tabular}

Table 5 Features of hepatic inflammation and steatosis

\begin{tabular}{llll}
\hline & Mild $(n / N)$ & Moderate $(n / N)$ & Severe $(n / N)$ \\
\hline Inflammation & & & \\
$\quad$ Portal & $48 / 98$ & $9 / 98$ & $3 / 98$ \\
$\quad$ Lobular & $2 / 98$ & $0 / 98$ & $0 / 98$ \\
$\quad$ Portal + lobular & $17 / 98$ & $5 / 98$ & $0 / 98$ \\
Macrovesicular steatosis & & & \\
$\quad$ Centrilobular & $28 / 98$ & $9 / 98$ & $0 / 98$ \\
$\quad$ Panaciner & $8 / 98$ & $2 / 98$ & $2 / 98$ \\
Microvesicular steatosis & $3 / 98$ & $0 / 98$ & $0 / 98$ \\
\hline
\end{tabular}

in the stomach, small intestine, and large intestine. Bleeding was observed in the gastric and small intestinal mucosae (Fig. 6).

The prostate was sampled with suspicion of hyperplasia during an autopsy in one case. Benign prostatic hyperplasia and acute prostatitis were observed in microscopic examination (Fig. 7).

\section{Discussion}

Our study offers a systematic overview of 100 cases infected with SARS-CoV-2 in Turkey. To the best of our knowledge, this is the largest review series performed in a single-center where by three body cavities were opened under a systematic approach in which organs and tissues are examined in detail. Autopsy findings are crucial to understand how this infection

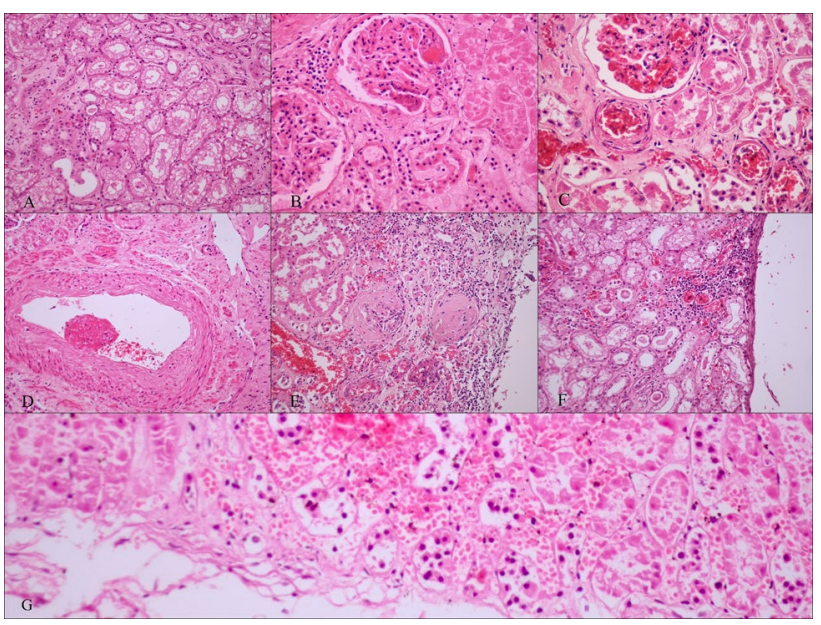

Fig. 3 A Acute tubular damage, $\times$ 200; B Glomerular capillary microvascular thrombosis, $\times 400$; C Glomeruler and peritubular capillary congestion, $\times 400$; $\mathbf{D}$ Fibrin trombosis in arcuate branch of the renal artery, $\times 200$; E Chronic pyelonephritis, $\times 200$; F Acute tubular damage, capillary endoteliitis, $\times 200$; G Subcapsular microhemorrhage, autolytic changes, $\times 100, \mathrm{H} \& \mathrm{E}$ 
Table 6 Renal pathological findings

\begin{tabular}{|c|c|c|c|}
\hline Histopathology & $n / N$ & Histopathology & $n / N$ \\
\hline Chronic pyelonephritis & $37 / 100$ & $\begin{array}{l}\text { Peritubular and glomerular } \\
\text { Capillary congestion }\end{array}$ & $81 / 100$ \\
\hline Tubular damage & $36 / 100$ & $\begin{array}{l}\text { Subcapsular microhemor- } \\
\text { rhage }\end{array}$ & $16 / 100$ \\
\hline $\begin{array}{l}\text { Interstitial lympho- } \\
\text { cytic inflammation }\end{array}$ & $15 / 100$ & Endotheliitis & $11 / 100$ \\
\hline Tubulary damage & $36 / 100$ & $\begin{array}{l}\text { Microvascular fibrin } \\
\text { thrombosis }\end{array}$ & $9 / 100$ \\
\hline Acute tubular necrosis & $3 / 100$ & Arcuate artery thrombosis & $1 / 100$ \\
\hline
\end{tabular}

affects the human body, as with other infectious diseases. Postmortem studies play a role not only in recognizing and understanding the disease, but also in determining the treatment methods to be applied for the disease.

After COVID-19 started to spread all over the world, nasopharyngeal, tracheal, and lung swab samples were taken in autopsy cases with suspect of COVID-19 in our department and PCR test for SARS-CoV-2 was started. Autopsies of the decedents with positive PCR test results were performed in the negative pressure autopsy room mostly on the same day. As far as we know, the largest single center published, with 80 cases, belongs to Edler et al. In this study, autopsies were performed within an average of 4 days after death. Histopathological data are limited in their studies [12]. In our autopsy cases, we tried to prevent autolysis by minimizing the time between PCR sampling and autopsy. In this way, it is aimed to examine pathological changes in organs with a better quality.

COVID-19 primarily affects the respiratory system, but it has been reported that the cardiovascular system, urinary, gastrointestinal system, reproductive, and nervous systems are also involved. Data on pathological changes in extrapulmonary organs are limited [13]. SARS-CoV-2 has been detected mostly in extrapulmonary organs which include the heart, kidney, brain, gastrointestinal system, liver, spleen, bone marrow, lymph nodes, and blood vessels. The possibility of direct viral infection in the tissues increases when the viral load is high [14]. The lesions that may develop in the organs and the pathology of COVID-19 can also be explained by autopsy studies.

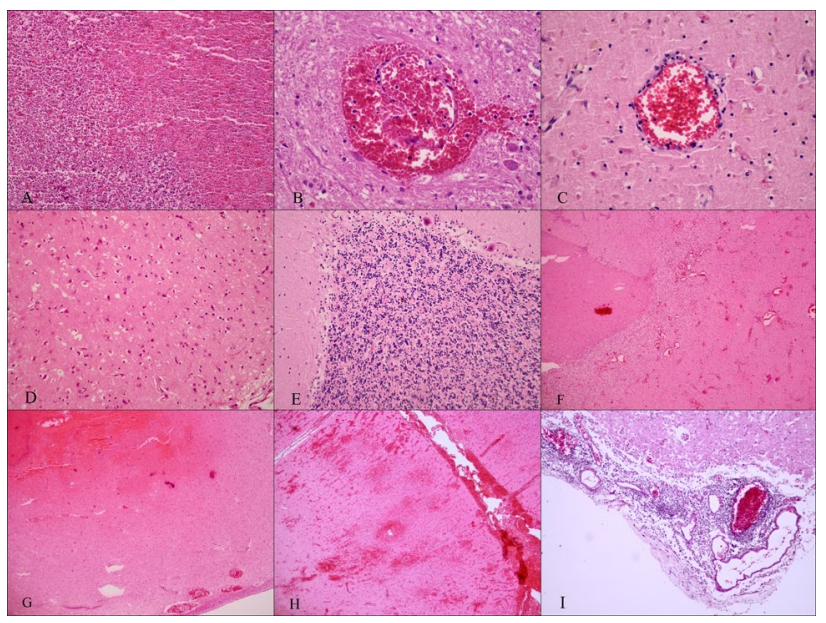

Fig. 4 A Pituitary necrosis, $\times 100$; B Perivascular petechial hemorrhage, $\times$ 400; C Capillary endoteliitis, $\times$ 400; D Acute hypoxic ischemic changes in the brain, $\times 200$; $\mathbf{E}$ Acute hypoxic ischemic changes in the cerebellum, $\times 200 ; \mathbf{F}$ Subacute infarction, $\times 40 ; \mathbf{G}$ Intraparenchymal and petechial hemorrhage, $\times 40$; H Microhemorrhages in infarct area, subarachnoid, $\times 40$, I Menengitis, $\times 100, \mathrm{H} \& \mathrm{E}$

It has been shown that there are similar numbers of cases in women and men but the mortality rate is higher in men [15]. Similarly, in our study, the male gender is dominant. The reasons for the high mortality in men may be higher levels of ACE-2 receptors in men, higher incidence of comorbid diseases in men, lower $\mathrm{CD} 3+, \mathrm{CD} 4+$ cell counts, $\mathrm{CD} 4+1$ $\mathrm{CD} 8+$ ratio, and $\mathrm{T}$ helper cell numbers in immune response compared to women [16]. The average age was found to be 79 in the study of Edler et al., 76 in the study of Menter et al., and 70 in the study of Satturwar et al. [12, 14, 17]. In our study, the average age is 54 , but the number of cases in the seventh decade is the highest. The fact that deaths accompanied by SARS-CoV-2 positivity such as drowning in water and traumatic deaths were included in the study, and the demographic and sociocultural structure of our country may be the reasons for the lower average age compared to other studies.

Regarding the places of death, in the Edler et al. study, $64 \%$ of the cases were reported as death in the hospital, $15 \%$ at home, and $16 \%$ in the nursing home [12]. In our study, $35 \%(n=35)$ of them were found dead at home. About $25 \%$
Table 7 Central nervous system pathological findings

\begin{tabular}{llll}
\hline Histopathology & $n / N$ & Histopathology & $n / N$ \\
\hline Perivascular petechial hemorrhage & $56 / 100$ & Meningoencephalitis & $3 / 100$ \\
Acute hypoxic/ischemic changes & $54 / 100$ & Subarachnoid hemorrhage & $11 / 100$ \\
Endotheliitis & $52 / 100$ & Intracranial hemorrhage & $9 / 100$ \\
Infarction & $8 / 100$ & Parenchymal microhemorrhage & $3 / 100$ \\
Microvascular fibrin thrombosis & $3 / 100$ & Intra-infarct hemorrhage & $2 / 100$ \\
\hline
\end{tabular}




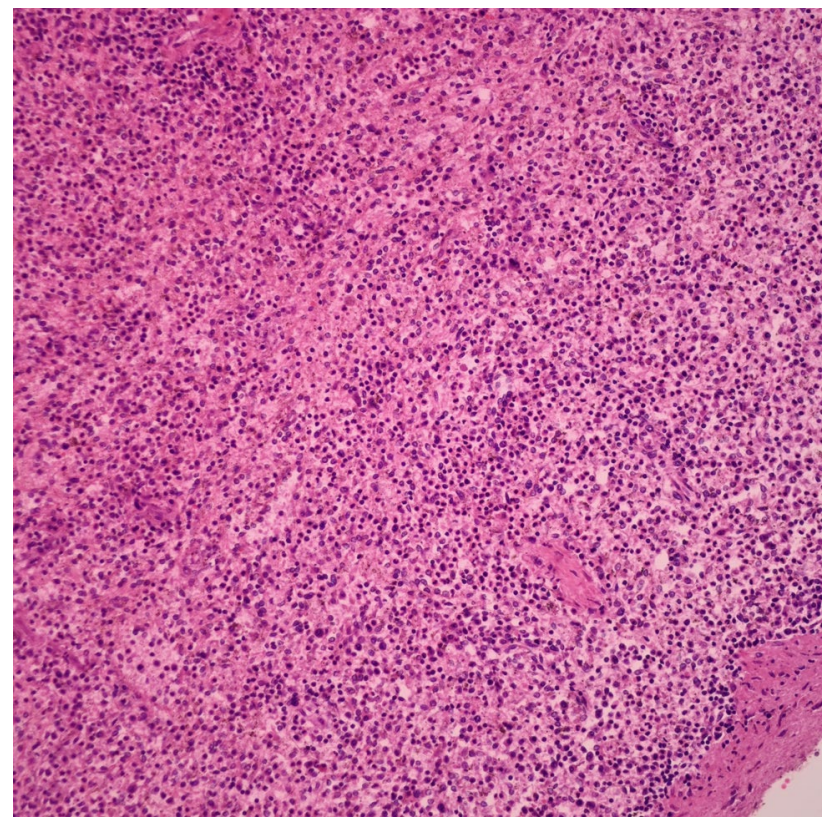

Fig. 5 Lymphocytic depletion and PMN leukocyte discharge in red pulp of the spleen, $\times 200, H \& E$

( $n=25)$ was death in hospital; 20\% $(n=20)$ was sudden death after illness. The presence of accompanying ischemic heart disease in most of the cases, the curfew restrictions of people over the age of 65 in our country, the patients having concerns about the risk of SARS-CoV-2 transmission, and therefore hesitating to go to the hospital even if they have a complaint, or being in quarantine at home may have increased the rate of being found dead at home and sudden deaths.

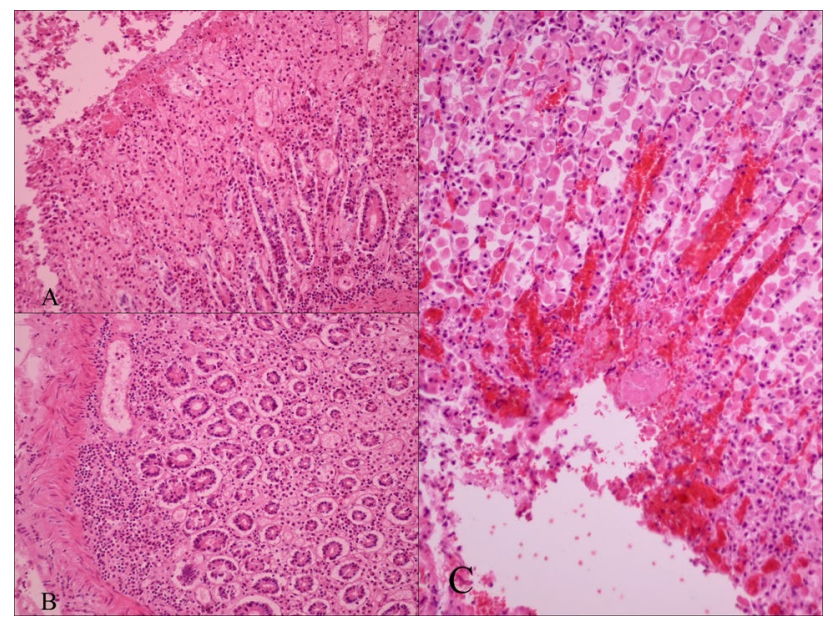

Fig. 6 A Necrotic changes in the epithelium of the small intestine, membrane-like fibrinous exudate on the mucosa, $\times 200$; B Perivascular lymphocytic mononuclear inflammatory cells, $\times 200$; C Necrotic changes in the epithelium of the stomach, membrane-like fibrinous exudate on the mucosa, mucosal hemorrhage, $\times 200, \mathrm{H} \& \mathrm{E}$

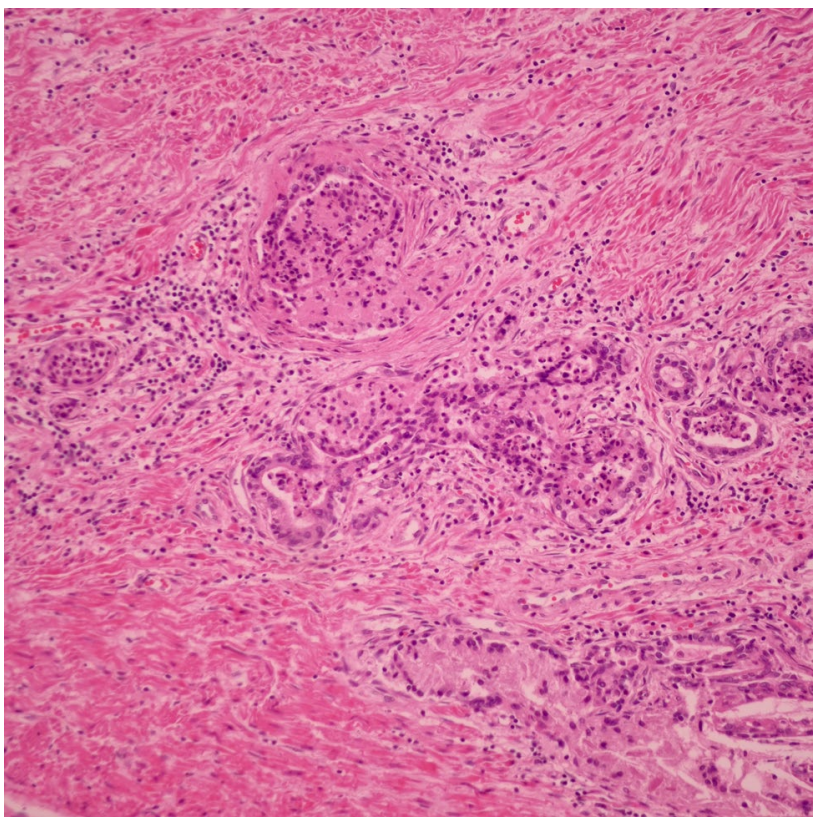

Fig. 7 Acut prostatitis, $\times 200, H \& E$

The heart is one of the important organs affected by SARS-CoV-2. Studies have shown myocyte necrosis, lymphocytic and eosinophilic myocarditis, and interstitial and epicardial mononuclear inflammatory cell infiltrates [18-20]. The mechanism of myocardial injury that develops in COVID-19 patients has not yet been clarified. It is thought that alveolar macrophages carrying SARS-CoV-2 particles migrate to the heart and cause direct injury, and the immune mechanisms of cardiac macrophages increase the possibility of rupture of the atheroma plaque and accelerate thrombus formation. Cardiac stress develops as a result of increased oxygen demand of the myocardium with fever and tachycardia and decreased oxygenation in the lung. Prolonged bed rest, increased fibrinogen production as a result of hepatic dysfunction, and inflammation contribute to myocyte injury by causing impairment in hemostasis. Systemic inflammation that develops as a result of cytokine storm indirectly causes injury to the myocardium [21-23].

In our study, ischemic lesions in the heart (scar, acute myocardial infarction, perivascular, and interstitial fibrosis), lymphohistiocytic myocarditis, interstitial lymphocytes, lymphocytic pericarditis, subendocardial hemorrhage, and amyloidosis were observed. Widespread areas of scar tissue due to previous myocardial infarction were observed in the vast majority of cases. The presence of ischemic lesions can be explained by the presence of the stenotic coronary arteries with atheroma plaque and the complication of these plaques. The presence of atheroma plaques and endothelial damage and the thrombogenic character of the disease may have accelerated the formation of thrombosis in the 
coronary arteries. Acute myocardial infarction was observed in patients with fibrin thrombosis in their coronary arteries. Among the risk factors in terms of hypercoagulability, as stated in other studies, the predominant number of males in the cases we included in the study; the high average BMI and the high number of elderly patients are among the risk factors [24]. This increases the risk of thrombosis. However, there are also cases in which thrombosis is not accompanied. These lesions may have developed due to the direct effect or complication of the disease. Senile amyloidosis was observed in one case. Menter et al. found that amyloidosis was more common in COVID-19 autopsies in their study [17]. We currently do not have data on the incidence of postmortem senile amyloidosis in non-COVID-19 autopsies at our department. However, the low mean age of the cases in our study may be one of the reasons for the lower rate (1\%) of senile amyloidosis. With new studies to be conducted, data to support the literature on the frequency of amyloidosis in COVID-19 cases may be revealed.

Studies on lesions that develop in the liver due to SARS$\mathrm{CoV}-2$ are limited. The mechanisms underlying liver damage are unclear. The direct effects of the virus on hepatocytes, cholangiocytes, and endothelial cells; the indirect effect of systemic immune response on liver cells; changes due to perfusion damage; drug-related liver damage; and preexisting liver pathologies are thought to be the mechanisms that cause injury [25, 26]. ACE 2 receptor is expressed more in bile duct cells than endothelial cells and hepatocytes. It is also thought that liver damage that occurs in COVID-19 patients may be due to damage to bile duct cells [27]. In autopsy studies, steatosis, portal and periportal inflammation, lobular inflammation, cholestasis, endothelitis, hepatocyte degeneration, and necrosis are among the main lesions $[14,28]$. In an autopsy study, macrovesicular steatosis was observed in the panacinar pattern in most of the cases and the periportal pattern in some of the cases. They thought that macrovesicular steatosis may develop during the disease process due to the lack of relationship between the degree of steatosis and body mass index, diabetes, drug use, and the distribution pattern of steatosis [29]. In postmortem biopsies, mild portal, and lobular inflammation, moderate steatosis were observed, and it was thought that the damage to the liver could be caused by drug-induced or SARS-CoV-2 infection [30].

Centrilobular mild macrovesicular steatosis and mild portal inflammation were the most common findings in our study. Steatosis is also one of the common findings in other studies in the literature [29, 31]. Centrilobular macrovesicular steatosis may also be associated with increased BMI, diabetes, and drug use in cases. It can be thought that macrovesicular steatosis in the panacinar pattern in $14 \%$ $(n=14)$ of the cases may have developed during the course of COVID-19 disease, based on the findings described in the literature. In our study, no statistically significant difference was found in cases with steatosis according to hospitalization. Therefore, no comment could be made on the possible relationship between steatosis and drug use. The precise pathophysiology of steatosis in the liver can be revealed by studies including clinical and laboratory findings. When the inflammation in the liver was evaluated in our cases, a lymphocytic inflammation rich in CD3 (+) lymphocytes was observed. Mild portal inflammation is the most common change. Less frequently, mild to moderate lobular inflammation accompanying portal inflammation was observed in some cases. Cholestasis is another noticeable finding. This may have arisen due to cholangiocytes being affected by SARS-CoV-2. It has been shown that SARS-CoV-2 affects the connections between cells and disrupts the structure of genes that regulate bile transport between cells [28]. Centrilobular necrosis seen in 9\% $(n=9)$ of the cases can be considered as an expected condition due to existing cardiovascular diseases.

The expression of ACE-2 receptors in tubular epithelial cells, endothelial cells, and podocytes has made the kidney a target for SARS-CoV-2 infection [19]. Acute tubular injury, which is an important condition that causes rapid clinical deterioration in COVID-19 cases and contributes to mortality, is the main pathological lesion [14]. Tubular damage is one of the striking lesions in our study, similar to the literature. Chronic pyelonephritis can also be considered as an expected condition due to comorbid diseases present in the cases. Microvascular thrombosis in glomerular capillary vessels has been associated with disseminated intravascular coagulation and generalized shock [17]. Microvascular thrombus in glomerular capillaries was seen in 9\% $(n=9)$ of the cases included in the study. Two of these cases were hospitalized, and the use of anticoagulants is unknown. In one case, fibrin thrombosis was observed in the renal artery branch. The thrombi in these cases may be thought to be caused by hypercoagulation as a result of the complication of the virus. Subcapsular microhemorrhage was a new and unexpected finding that attracted attention in $16 \%(n=16)$ of the cases. To the best of our knowledge, no information on subcapsular hemorrhage could be found in other studies. We think that this hemorrhage may have developed as a result of vascular damage due to the effect of the disease. With new studies, the relationship of this situation with COVID19 can be revealed. Similar to the findings in the literature, interstitial lymphocytic inflammation was observed in the subcapsular areas in our cases. Erythrocyte aggregates in capillary vessels, which we define as peritubular and glomerular capillary congestion, was observed in $80 \%(n=80)$ of the cases. It was found that this aggregate does not contain thrombocyte, fibrin, and fibrinoid necrosis, and it was thought to be associated with long-term hypotension [32]. 
Studies on pathological findings of central nervous system involvement in patients infected with SARS-CoV-2 are limited. Studies have shown that SARS-CoV-2 causes tissue damage in the olfactory nerve, gyrus rectus, and brain stem [33]. Changes related to thrombosis, acute infarction, petechial hemorrhage, and hypoxia/ischemia are among the pathologies seen in COVID-19 cases. This demonstrates the role of endothelial dysfunction and thrombosis in the neurological manifestations of COVID-19. The most common finding in autopsy studies is hypoxic changes. Solomon et al. found acute hypoxic damage in the brain and cerebellum in all cases in a series of 18 cases $[14,34]$. In our study, $54 \%(n=54)$ of the cases had acute hypoxic/ ischemic changes. However, these findings are nonspecific findings that cannot be associated with COVID-19. In our study, perivascular petechial hemorrhage in the white matter, brainstem, and cerebellum was another remarkable finding in $56 \%(n=56)$ of the cases. In addition, intraparenchymal microhemorrhage was observed in $3 \%$ $(n=3)$ of the cases. In $2 \%(n=2)$ of the cases, intra-infarct hemorrhage was observed. These hemorrhages are thought to be caused by reperfusion injury and vascular damage [35]. Subarachnoid and intraparenchymal hemorrhage were present in cases with SARS-CoV-2 positivity, who had a history of trauma such as falling from a height. In $1 \%$ $(n=1)$ of the cases, non-traumatic pathological cerebral hemorrhage developed. The vascular damage caused by the virus, history of trauma, and use of anticoagulant agents in the treatment of COVID-19 patients may have contributed to the more frequent occurrence of these hemorrhages and increased mortality. Until now, no direct virus-related meningoencephalitis cases have been reported in postmortem examinations. In the study of Rechard et al., parainfectious complications that may develop due to immune dysregulation were emphasized [36]. We think that meningoencephalitis, which we detected in $3 \%(n=3)$ of the cases, was a complication that developed during hospitalization as a result of traumatic hemorrhage and was not related to the direct effect of the virus.

Spleen is one of the hematopoietic organs affected by SARS-CoV-2. In many studies, findings such as the depletion of lymphocytes in the white pulp in the spleen, an increase in the ratio of red/white pulp, an increase in neutrophils in the red pulp, septic splenitis, and splenic infarction were found $[14,17,19]$. The depletion of lymphocytes in white pulp explains the clinically common lymphopenia in COVID-19 cases [14]. In our study, the spleen was sampled in 17 cases. In 16 of these cases, a decrease in CD3 (+) T lymphocytes was observed in the white pulp. A decrease in CD20 (+) B lymphocytes was observed in both red and white pulps. Also, in accordance with other studies in the literature, an increase in neutrophils was observed in the red pulp.
The expression of ACE- 2 receptors in islet cells and exocrine pancreas makes the pancreas one of the target organs for SARS-CoV-2. However, pancreatitis may develop with secondary immune-mediated damage during SARS-CoV-2 infection [37]. In our study, the pancreas was sampled in 21 cases, and focal acute pancreatitis was observed in 5\% $(n=1)$ of these cases. Since the clinical history of the case is unknown, a definite interpretation could not be made that it developed directly due to the virus.

When the gastrointestinal system was evaluated, mesenteric ischemia, regeneration of the epithelium, necrosis, and lymphoplasmacytic infiltrations in the stomach and intestines were observed in COVID-19 cases [38, 39]. Necrotic mucosa associated with pseudomembrane has been reported in one case [40]. In our study, samples were taken from the gastric and small intestine mucosa because of hemorrhagic appearances. In this case, dense lymphoplasmacytic infiltration around the capillary vessels, membrane-like fibrinous exudate on the epithelial surface, necrotic changes in the epithelium, and acute inflammation findings were noted. In addition, hemorrhagic areas were seen in the mucosa. The presence of SARS-CoV-2 was confirmed by PCR in the small intestine, and it was thought that this change was caused by the virus.

ACE-2 receptors are expressed in many organs, especially in the lung, as well as in endothelial cells. Vascular endothelium plays a role in regulating vascular tone and maintaining vascular homeostasis. Dysfunction in the endothelium results in microvascular dysfunction. This may result in vasoconstriction-related ischemia, inflammation, and coagulation activation. Varga et al. found inflammation of endothelial cells in their study. They have shown that SARS-CoV-2 infection facilitates the development of endotheliitis in many organs [39, 41, 42]. In our study, findings of endotheliitis were observed in many organs. Capillary endothelitiis was seen in the liver and kidney, especially in the central nervous system. It was observed that this inflammation in the endothelium was rich in CD3 (+) T lymphocytes. In addition, microvascular fibrin thrombi were noted in these organs. We think that these thrombi developed as a result of endothelial dysfunction.

Our study has some limitations. PCR could not be performed in all tissues, and immunohistochemical staining for $\mathrm{CD} 3$ and CD20 was studied in a limited number of cases. Electron microscopic evaluation could not be done. The high number of death in home and sudden death cases, postmortem incidental detection of SARS-CoV-2 positivity in some cases, insufficient information from relatives of the deceased, unknown duration of antemortem SARS-CoV-2 positivity, unknown history of drug use, and insufficient information about existing comorbid diseases are also among the limitations of our study. In our institution, PCR results are obtained within the same day after 
sampling, and for this reason, autopsies were mostly performed on the same day in our cases to prevent autolysis.

In some of the cases, the swab result was either negative or no samples were taken for PCR prior to autopsy. During the histopathological examination, we detected positivity by performing a PCR test from paraffin blocks from the cases we found suspicious for COVID-19 in the organs.

\section{Conclusion}

Our study has evaluated the changes of SARS-CoV-2 in extrapulmonary organs unlike many studies which have mostly focused on pulmonary changes. We believe that our study results will contribute to understand the pathophysiology of SARSCoV-2 in extrapulmonary organs. We have shown how autopsies plays an important role in systematically examining the damage caused by the virus in all organs in order to elucidate the pathogenesis of SARS-CoV-2 infection and contribute to the clinical management of infected patients. We hope that these findings will contribute to a better understanding of SARS-CoV-2 and development of new treatment strategies.

\section{Key points}

1. SARS-CoV-2 is a new enveloped RNA virus that causes COVID-19 disease that causes pandemic in all over the world.

2. Although SARS-CoV-2 infection primarily affects the lung, it turns into viral sepsis and causes hypercoagulation and multiorgan failure.

3. Autopsies of people who died from the SARS-CoV-2 infection can provide important information about the new disease and its course.

4. COVID-19 primarily affects the respiratory system, but cardiovascular system, urinary, gastrointestinal system, reproductive, and nervous systems are also involved.

Acknowledgements I thank the Council of Forensic Medicine for allowing us to do this work and for their support. I thank Dr. Abdul Kerim Buğra for his support for the statistical analysis of our study. Thank you to our staff in our Pathology Laboratory.

Author Contribution All authors equally contributed to the study.

\section{Declarations}

Ethics approval This study was carried out with the approval of the Council of Forensic Medicine Education and Scientific Research Commission. (21589509/2020/1122).

\section{References}

1. Zhu N, Zhang D, Wang W et al (2020) A novel coronavirus from patients with pneumonia in China 2019. N Engl J Med 382(8):727-733

2. WHO Coronavirus Disease Dashboard. https://covid19.who.int/. Accessed 1 Feb 2021

3. Republic of Turkey Ministry of Health. Covid-19 Information Page. https://covid19.saglik.gov.tr/. Accessed 1 Feb 2021

4. de Groot RJ, Baker S, Baric R et al (2012) Family coronaviridae. In: King AMQ, Adams MJ, Cartens EB, Le owitz EJ (eds.), Virus taxonomy, the 9th report of the International Committee on Taxonomy of Viruses. Academic Press, San Diego, CA. https://doi.org/10.1016/B978-0-12-384684-6.00068-9 p. 806-828

5. Pillaiyar T, Meenakshisundaram S, Manickam M (2020) Recent discovery and development of inhibitors targeting coronaviruses. Drug Discov Today. https://doi.org/10.1016/j.drudis.2020.01.015

6. Mahase E (2020) Coronavirus: covid-19 has killed more people than SARS and MERS combined, despite lower case fatality rate. BMJ 368:m641. https://doi.org/10.1136/bmj.m641

7. Guan WJ, Ni ZY, Hu Y et al (2020) Clinical characteristics of coronavirus disease 2019 in China. N Engl J Med 382(18): 1708-1720

8. Tiwari R, Dhama K, Sharun K et al (2020) COVID-19: animals, veterinary and zoonotic links. Vet Q 40(1):169-182

9. Wu A, Peng Y, Huang B et al (2020) Genome composition and divergence of the novel coronavirus (2019-nCoV) originating in China. Cell Host Microbe 27(3):325-328

10. Hamming I, Timens W, Bulthuis ML et al (2004) Tissue distribution of ACE2 protein, the functional receptor for SARS coronavirus. A first step in understanding SARS pathogenesis. J Pathol 203(2):631-7

11. Maiese A, Manetti AC, La Russa R et al (2020) Autopsy findings in COVID-19-related deaths: a literature review. Forensic Sci Med Pathol 7:1-18. https://doi.org/10.1007/s12024-020-00310-8

12. Edler C, Schröder AS, Aepfelbacher M et al (2020) Dying with SARS-CoV-2 infection-an autopsy study of the first consecutive 80 cases in Hamburg, Germany. Int J Legal Med 134(4):12751284. https://doi.org/10.1007/s00414-020-02317-w. Epub 2020 Jun 4. Erratum in: Int J Legal Med 134(5):1977. PMID: 32500199; PMCID: PMC7271136.

13. UyarBozkurt S (2020) Neuropathology of COVID-19. Çelikel Ç. edits Pathology of COVID-19. 1st edit. Türkiye Klinikleri $1: 20-28$

14. Satturwar S, Fowkes M, Farver C et al (2021) Postmortem findings associated with SARS-CoV-2: systematic review and meta-analysis. Am J Surg Pathol. https://doi.org/10.1097/PAS 0000000000001650 . Epub ahead of print. PMID: 33481385.

15. Gebhard C, Regitz-Zagrosek V, Neuhauser HK et al (2020) Impact of sex and gender on COVID-19 outcomes in Europe. Biol Sex Differ 11:1-13

16. Jia HP, Look DC, Tan P et al (2009) Ectodomain shedding of angiotensin converting enzyme 2 in human airway epithelia. Am J Physiol Lung Cell Mol Physiol 297(1):L84-96

17. Menter T, Haslbauer JD, Neinhold R et al (2020) Post-mortem examinations of COVID-19 patients reveals diffuse alveolar damage with severe capillary congestion and variegated findings in lungs and other organs suggesting vascular dysfunction. Histopathology 77:198-209

18. Basso C, Leone O, Rizzo S et al (2020) Pathological features of COVID-19-associated myocardial injury: a mutlicentre cardiovascular pathology study. Eur Heart J 41:3827-3835

19. Bradley BT, Maioli H, Johnson R et al (2020) Histopathology and ultrastructural findings of fatal COVID-19 infections in Washington State: a case series. Lancet 396:320-332 
20. Craver R, Huber S, Sandomirsky M et al (2020) Fatal eosinophilic myocarditis in a healthy 17-year-old male with severe acute respiratory syndrome coronavirus 2 (SARS-CoV-2c). Fetal Pediatr Pathol 39:263-268

21. Hatami F, Valizadeh N, Ansari Ramandi MM (2020) Emerging mechanisms for the new coronavirus-related myocardial injury and ischemia: A review of the literature. Anatol J Cardiol 24(1):7-12

22. Tavazzi G, Pellegrini C, Maurelli M et al (2020) Myocardial localization of coronavirus in COVID-19 cardiogenic shock. Eur J Heart Fail 22:911-915

23. Libby P, Loscalzo J, Ridker PM et al (2018) Inflammation, immunity, and infection in atherothrombosis: JACC Review Topic of the Week. J Am Coll Cardiol 72:2071-2081

24. Yamamoto K, Takeshita K, Kojima T et al (2005) Aging and plasminogen activator inhibitor-1 (PAI-1) regulation: implication in the pathogenesis of thrombotic disorders in the elderly. Cardiovasc Res 66:276-285

25. Abenavoli L, Gentile I, Maraolo AE, Negro F (2020) SARS-CoV-2 and liver damage: a possible pathogenetic link. HepatoBiliary Surg Nutr 9(3):322-324

26. Alqahtani SA, Schattenberg MJ (2020) Liver injury in COVID-19: the current evidence. United European Gastroenterology Journal 8(5):509-519

27. Xu L, Liu J, Lu M et al (2020) Liver injury during highly pathogenic human coronavirus infections. Liver Int 40:998-1004

28. Çelikel Ç (2020) COVID-19-gastrointestinal kanal ve karaciğer patolojisi. Türkiye Klinikleri 1:29-38

29. Lagana SM, Kudose S, Iuga AC et al (2020) Hepatic pathology in patients dying of COVID-19: a series of 40 cases including clinical, histologic, and virologic data. Mod Pathol 33(11):2147-2155

30. Xu Z, Shi L, Wang Y et al (2020) Pathological findings of COVID-19 associated with acute respiratory distress syndrome. Lancet Respir Med. https://doi.org/10.1016/S2213-2600(20) 30076-X

31. Martines RB, Ritter JM, Matkovic E et al (2020) COVID-19 Pathology Working Group. Pathology and pathogenesis of SARSCoV-2 associated with fatal coronavirus disease, United States. Emerg Infect Dis 26(9):2005-2015. https://doi.org/10.3201/ eid2609.202095. Epub 2020 May 21. PMID: 32437316; PMCID: PMC7454055.
32. Su H, Yang M, Wan C et al (2020) Renal histopathological analysis of 26 postmortem findings of patients with COVID- 19 in China. Kidney Int 98(1):219-227

33. Bulfamante G, Chiumello D, Canevini MP et al (2020) First ultrastructural autoptic findings of SARS-Cov-2 in olfactory pathways and brainstem. Minerva Anestesiol 86(6):678-679

34. Solomon IH, Normandin E, Bhattacharyya S et al (2020) Neuropathological features of Covid-19. N Engl J Med [Epub ahead of print]

35. Bryce C, Grimes Z, Pujadas E et al (2020) Pathophysiology of SARS-CoV-2: targeting of endothelial cells renders a complex disease with thrombotic microangiopathy and aberrant immune response. The Mount Sinai COVID-19 autopsy experience. medRxiv 2020.05.18.20099960

36. Reichard RR, Kashani KB, Boire NA et al (2020) Neuropathology of COVID-19: a spectrum of vascular and acute disseminated encephalomyelitis (ADEM)-like pathology. Acta Neuropathol 140:1-6

37. Samies NL, Yarbrough A, Boppana S (2021) Pancreatitis in pediatric patients with COVID-19. J Pediatric Infect Dis Soc 10(1):57-59. https://doi.org/10.1093/jpids/piaa125

38. Xiaohong Y, Tingyuan L, Zhicheng H et al (2020) New coronavirus pneumonia (COVID-19) 3 cases of multiple-site puncture histopathological pathology of human remains. Chin J Pathol 49:291-293

39. Varga Z, Flammer AJ, Steiger P et al (2020) Endothelial cell infection and endotheliitis in COVID-19. Lancet 395:1417-1418

40. Wichmann D, Sperhake JP, Lütgehetmann M et al (2020) Autopsy findings and venous thromboembolism in patients with COVID19. Ann Intern Med 173:268-277

41. Flammer AJ, Anderson T, Celermajer DS et al (2012) The assessment of endothelial function: from research into clinical practice. Circulation 126(753-67):6

42. Bonetti PO, Lerman LO, Lerman A (2003) Endothelial dysfunction-a marker of atherosclerotic risk. Arterioscl Throm Vas 23:168-175

Publisher's Note Springer Nature remains neutral with regard to jurisdictional claims in published maps and institutional affiliations. 